\title{
H. Lanfermann, P. Raab, H-J Kretschmann, and W. Weinrich (Editors): Cranial neuroimaging and clinical neuroanatomy (fourth edition)
}

\author{
P Georg Thieme Verlag KG: Stuttgart 2019, 513 pp., 930 figures ISBN 978-3-13-672604-4
}

\author{
Mario Giordano ${ }^{1}$ \\ Received: 15 March 2020 / Accepted: 23 March 2020 / Published online: 8 May 2020 \\ (C) Springer-Verlag GmbH Germany, part of Springer Nature 2020
}

The academic anatomical and neurosurgical literature has made enormous progresses in last decades. Indeed, the introduction of new investigation technology resulted not only in better understanding of the human nervous system but also impacted on the traditional way of teaching.

In this scenario, the book here considered "Cranial neuroimaging and clinical neuroanatomy" edited by $\mathrm{H}$. Lanfermann, P. Raab, H-J Kretschmann, and W. Weinrich can be to a certain extent regarded a precursor work that continues to be constantly reinvented.

This new fourth edition conveys the above-mentioned concepts. It provides a further insight into the complex topic of nervous system anatomy with the support of modern artworks and neuroradiology imaging and adds to the classical anatomical descriptions new acquisitions, namely in the sections devoted to the brain development and to the neurofunctional system.

The volume has 513 pages and an enormous number of illustrations including anatomical drawings, MRI images, and comparison of both in order to identify the anatomical structures on MRI. Particularly didactic are the figures obtained from 35 fixed brains dissected at the Hannover Medical School with the corresponding sectional artist drawings that explain the anatomical structure in a very accessible fashion.

The book is well organized with its practical subdivision in four main sections: introduction, Atlas, Topography of head and neck, and Neurofunctional Systems.

It is composed by 11 different chapters (two, four, three and two for section one to four, respectively) and a separated appendix containing information about the preparation of

Mario Giordano

Giordano.nch@gmail.com; mario.giordano@alice.it

1 International Neuroscience Institute Hannover $\mathrm{GmbH}$, Hannover, Germany specimens and techniques. Of great utility are the comparison of artist drawing with the same structures in MRI and CT scan in all three planes that are shown in section number 2 (Atlas) of the book with explicative legends and complete description of the anatomy.

The part three is mainly composed by topographic anatomy of the head and neck with particular attention to the intracranial space and the different vascular territories. In this section, also the physiology and clinical significance of the structures are briefly discussed. The neurofunctional systems with a subsection dedicated to neurotransmitters and neuronal networks are fully described in section four: this is one of the most interesting part that connects the anatomy with the functions of the structures.

In comparison with previous editions, new parts regarding brain maturation and temporal bone anatomy have been included. Moreover, the sections about cranial vessels, with the addition of the arterial territories of the infratentorial space, have been improved. Also, the illustrative drawings and explanation of neurofunctional systems have been enhanced.

The authors, who are leaders in the field, should be praised for their detailed and easy to understand explanations of neuroanatomical and neuroradiological images. An important mention should be given to the quality of printing and to the rich iconography which characterizes Thieme medical publications. The book provides neurosurgery, neurology, and neuroradiology residents an extraordinary educational tool to improve their anatomical knowledge. It can be also of great aid for specialists in their everyday practice.

\section{Compliance with ethical standards}

Conflict of interest None.

Publisher's note Springer Nature remains neutral with regard to jurisdictional claims in published maps and institutional affiliations. 